\title{
Germanica
}

\section{Le cinéma entre totalitarisme et démocratie}

Olivier AGARD : Das Kino zwischen Totalitarismus und Demokratie

Olivier AGARD : The cinema between totalitarianism and democratie

\section{Olivier Agard}

\section{(QpenEdition \\ Journals}

\section{Édition électronique}

URL : https://journals.openedition.org/germanica/8506

DOI : $10.4000 /$ germanica. 8506

ISSN : 2107-0784

\section{Éditeur}

Université de Lille

Édition imprimée

Date de publication : 1 juin 2020

Pagination : 15-30

ISBN : 978-2-913857-45-2

ISSN : 0984-2632

\section{Référence électronique}

Olivier Agard, «Le cinéma entre totalitarisme et démocratie », Germanica [En ligne], 66 | 2ème trimestre 2020, mis en ligne le 02 janvier 2022, consulté le 07 janvier 2022. URL : http://

journals.openedition.org/germanica/8506 ; DOI : https://doi.org/10.4000/germanica.8506

\section{(c) Tous droits réservés}




\title{
Le cinéma entre totalitarisme et démocratie
}

\author{
Olivier Agard \\ Sorbonne-Université
}

Les analyses de Kracauer sur la propagande, maintenant éditées dans le volume 2 des œuvres complètes ${ }^{1}$, sont intéressantes à plusieurs titres : tout d'abord, elles documentent le développement de la pensée de Kracauer dans l'exil. En effet, elles s'inscrivent à certains égards dans la continuité des feuilletons des années 1920, tout en posant aussi les bases des écrits américains de Kracauer : From Caligari to Hitler $(1947)^{2}$, Theory of film $(1960)^{3}$, History: The last things before the last (parution posthume en 1969) 4 . À l'intérieur de cet ensemble de textes, on

1. - Siegfried Kracauer, Studien zu Massenmedien und Propaganda (hg. v. Christian Fleck und Bernd Stiegler; unter Mitarbeit von Joachim Heck und Maren Neumann) [Werke; 2.2], Frankfurt a.M., Suhrkamp, 2012.

2. - Siegfried Kracauer, From Caligari to Hitler, a psychological history of the German film, London, D. Dobson ; New York, the Riverside press, 1947. Traduction française : S. Kracauer, De Caligari à Hitler : une histoire psychologique du cinéma allemand (traduit de l'anglais par Claude B. Levenson; avec une postface de Leonardo Quaresima), Paris, L'Âge d'homme, 2009.

3. - Siegfried Kracauer, Theory of film: the redemption of physical reality, London et al., Oxford University press, 1960. Traduction française : S. Kracauer, Théorie du film : la rédemption de la réalité matérielle (traduit de l'anglais par Daniel Blanchard et Claude Orsoni ; édité et présenté par Philippe Despoix et Nia Perivolaropoulou ; préface de Jean-Louis Leutrat), Paris, Flammarion, 2010.

4. - Siegfried Kracauer, History: The last things before the last (foreword by Paul Oscar Kristeller), New York, Oxford University press, 1969. Traduction française : S. Kracauer, L'histoire : des avant-dernières choses (traduit de l'anglais par Claude 
peut distinguer me semble-t-il deux moments : dans un premier temps, dans le long essai sur la propagande totalitaire de 1937-38 (reconstitué par Bernd Stiegler et ses collaborateurs ${ }^{5}$ ), Kracauer se penche plutôt sur la fonction générale de la propagande dans le système national-socialiste et fasciste. L'accent porte alors sur une analyse du «totalitarisme » comme dispositif politique : on est dans le domaine de la philosophie politique, et Kracauer semble peu s'intéresser aux contenus concrets de la propagande, qu'il ne connaissait d'ailleurs pas de première main. Dans un second groupe de texte rédigés aux États-Unis, Kracauer se concentre en revanche sur ces matériaux concrets, principalement les actualités cinématographiques nazies, et les films montés à partir d'elles, comme Sieg im Westen (1941) ou Feuertaufe (1940). On est alors davantage sur le terrain d'une analyse esthétique. Cette différence d'approche et de méthode s'explique par le contexte européen, puis américain, dans lequel ces travaux ont respectivement vu le jour. Dans les deux cas, il s'agit en effet de travaux de commande, dont Kracauer, comme beaucoup d'exilés, était dépendant pour sa survie. Il n'est pas certain que l'intérêt profond de Kracauer se portait alors sur l'analyse de la propagande : derrière cette analyse se profile déjà sa réflexion théorique sur le cinéma, qui aboutira à la Théorie du film en 1960. Après la publication de la biographie de Jacques Offenbach en 1937 (Jacques Offenbach ou le secret du Second Empire ${ }^{6}$ ), son projet principal semble avoir été longtemps celui d'une histoire socio-culturelle du cinéma mondial. Si l'on en croit une lettre à Mme Viénot, il s'agissait d'un travail à vocation sociohistorique ${ }^{7}$. En mai 1938, il présente le livre comme une sorte d'histoire culturelle du passé récent ${ }^{8}$ et annonce une série de chapitres (le Slapstick américain, la période du Jazz, l'influence de la crise économique, la production de films de divertissement, Hollywood). Dans une demande de bourse à l'American Guild, l'ouvrage est décrit par Kracauer comme « un livre sur le cinéma, dans lequel je veux montrer sa signification sociologique, esthétique et socioculturelle de 1895 à nos jours ${ }^{9} »$. Pour

Orsoni ; édité par Nia Perivolaropoulou et Philippe Despoix ; présentation de Jacques Revel), Paris, Stock, 2006.

5. - S. Kracauer, Studien zu Massenmedien und Propaganda, op. cit., p. 17173. Voir également Siegfried Kracauer, Totalitäre Propaganda (hg. und mit einem Nachwort versehen von Bernd Stiegler; unter Mitarbeit von Joachim Heck und Maren Neumann), Berlin, Suhrkamp, 2013.

6. - Siegfried Kracauer, « Marseiller Entwurf», in: Siegfried Kracauer, Theorie des Films: die Errettung der äusseren Wirklichkeit: mit einem Anhang "Marseiller Entwurf" zu einer Theorie des Films [Werke 3], Frankfurt a.M., Suhrkamp, 2005, p. 521-803.

7. - Lettre de Kracauer à Mme Viénot du 30.5.1937, Kracauer Nachlass, Deutsches Literaturarchiv (KN DLA).

8. - Siegfried Kracauer, "Plan meines Buches über den Film », daté du 16 mai 1938, KN DLA.

9. - Werner Berthold, Britta Eckert, Franck Wende, Deutsche Intellektuelle im 
préparer ce livre, il passa les six derniers mois de 1938, l'année 1939 et les six premiers mois de 1940 au cinéma (à l'exception de deux mois entre septembre et novembre 1939, où il est cantonné dans des camps d'internement, à Maisons Lafitte puis Athis-sur-Orne), mettant inlassablement les films en fiches. Il suivit alors assidument les projections organisées par Henri Langlois et le « Cercle du cinéma ». Pour Kracauer, il s'agissait, contre le fascisme, de fonder la possibilité d'un accès du sujet à ce qu'on peut appeler la « réalité », dans une perception partagée qui était la condition de l'établissement d'une communauté démocratique. Kracauer est à la recherche d'une réponse productive au fascisme et à sa tendance à la déréalisation, et dès les années 1930, le cinéma joue selon lui dans ce contexte un rôle central, comme en témoigne notamment ce qu'on peut appeler sa première théorie du film, qui consiste en un manuscrit inachevé rédigé à Marseille en 194010.

\section{La propagande totalitaire}

Le premier grand travail de Kracauer sur la propagande, intitulé Die totalitäre Propaganda a vu le jour entre juillet 1937 et avril 1938, et c'est une commande de l'Institut de Recherche en Sciences Sociales (Institut für Sozialforschung). Lorsque ce travail est terminé, en avril 1938, l'Institut refuse sa publication en l'état. Adorno réduit et réécrit le texte ${ }^{11}$, et Kracauer s'oppose alors à la publication de cette version tronquée. Curieusement, dans ce texte, il n'est pas explicitement question de cinéma, ce qui s'explique sans doute par le fait que Kracauer n'avait pas d'accès direct aux contenus de la propagande en général et aux films allemands en particulier. Cette orientation s'explique aussi par le lieu de publication prévu et les attentes d'Adorno et Horkheimer. La Zeitschrift für Sozialforschung abordait en effet le fascisme sous l'angle d'une théorie du " capitalisme de monopole », utilisant les instruments de la critique de l'idéologie et la psychanalyse, mais sans s'appuyer vraiment sur des études de cas précises. Toutefois, même si le cinéma semble absent de Totalitäre Propagande, l'image n'est chez Kracauer jamais loin, et on peut lire ce travail sur la propagande comme un commentaire du Triomphe de la volonté (1935) de Leni Riefenstahl, ou du moins, ce que dit Kracauer s'applique assez bien à ce film, dont il avait certainement dû entendre parler, à défaut de l'avoir vu. Ce film avait en effet été montré

Exil: Ihre Akademie und die "American Guild for German Culture », München u.a., Saur, 1993, p. 307-313.

10. - Siegfried Kracauer, Jacques Offenbach und das Paris seiner Zeit, Amsterdam, Allert de Lange, 1937. Traduction française : S. Kracauer, Jacques Offenbach ou Le secret du Second Empire (préf. de Daniel Halévy ; trad. de l'allemand par Lucienne Astruc), Paris, le Promeneur-Gallimard, 1994 (initialement : Grasset 1937).

11. - Le texte se trouve dans le volume Totalitäre Propaganda, op. cit., p. 266-296. 
en 1937 à plusieurs reprises dans le cadre de l'Exposition Universelle, dans le pavillon allemand ${ }^{12}$, et même s'il n'a alors été vu que par un public restreint, il en a été question dans la presse, Leni Riefenstahl jouissant d'une grande notoriété en tant qu'" amazone du Troisième Reich ». Le film fut par ailleurs également projeté le 28 mai 1937 au palais de Chaillot, dans le cadre du "Cercle du cinéma » de Georges Franju et Henri Langlois, dont Kracauer était membre, comme on l'a dit ${ }^{13}$.

Au cœur de l'interprétation que Kracauer donne du national-socialisme dans Totalitäre Propaganda, il y a l'idée que le régime produit une pseudo-réalité pour dissimuler et désamorcer les antagonismes sociaux. Cette idée d'une pseudo-réalité, Kracauer l'emprunte en partie à Marx. Dans une note qui se trouve dans les brouillons du Jacques Offenbach, un ouvrage qui, de façon cryptée, est aussi un livre sur l'Allemagne nazie, Kracauer reproduit la célébrissime phrase de l'introduction du 18 Brumaire : « Hegel remarque quelque part que tous les grands faits et les grands personnages de l'histoire universelle adviennent pour ainsi dire deux fois. Il a oublié d'ajouter : la première fois comme tragédie, la seconde fois comme farce ${ }^{14}$ ». À côté de cette citation, Kracauer a écrit au crayon rouge : « Société d'opérette » («Operettengesellschaft 》). Pour établir et pérenniser son pouvoir, Louis Napoléon Bonaparte doit selon Kracauer produire l'illusion d'une réconciliation des classes sociales, et à cette fin, il promeut une culture festive d'éclat et de joie, supposée griser et éblouir (« D'où la lumière et la joie. La joie pour griser, la lumière pour éblouir $\left.{ }^{15} \gg\right)$. Offenbach s'est alors selon Kracauer coulé d'une façon ambiguë dans ce projet politique, en le subvertissant de l'intérieur. Dans la Propagande totalitaire, cette pseudo-réalité prend la forme d'une image, celle d'un peuple réconcilié et homogène. Ce thème d'un pseudopeuple en trompe-l'œil apparaissait déjà dans Jacques Offenbach, mais on était alors plutôt dans le domaine du théâtre. Kracauer avait en effet été frappé dans Le 18 Brumaire de Louis Bonaparte par la caractérisation de Louis Napoléon Bonaparte, « Napoléon III », en personnage qui « considère la vie des peuples, leur activité et celles de l'État, comme une comédie », et qui s'entoure lors de ses déplacements de figurants censés représenter le peuple ${ }^{16}$. Dans la Propagande totalitaire, ce pseudopeuple fait place à une simple image, celle d'une masse homogène. Pour la propagande totalitaire nazie, il s'agit de mettre en scène une

12. — Jérôme Bimbenet, Leni Riefenstahl : la cinéaste d'Hitler, Paris, Tallandier, 2015, p. 133-135.

13. - Ibid., p. 133.

14. - Karl Marx, Le dix-huit brumaire de Louis Bonaparte (traduction, présentation, notes, chronologie, bibliographie et index par Grégoire Chamayou), Paris, Flammarion, 2007, p. 49.

15. - S. Kracauer, Jacques Offenbach, op. cit., p. 140.

16. — Ibid., p. 99. 
communauté du peuple fictive, sous la forme d'une image sans réalité : on met alors au premier plan les concepts de nation et de peuple pour simuler une unité et on contraint la masse à se contempler elle-même comme un spectacle permanent. La masse n'est donc pas seulement un moyen de propagande, elle est elle-même propagande. Kracauer parle « d'une pseudo-intégration des masses artistiquement préparées par la propagande $^{17} \gg$. Cette image idéalisée d'une communauté du peuple fictive ressemble à ce que Benjamin décrit dans son essai sur L'œuvre d'art à l'époque de sa reproductibilité technique comme l'« esthétisation du politique ». Kracauer souligne lui aussi le caractère esthétique de la propagande, qu'il associe à son « nihilisme » :

Le même nihilisme qui conditionne le comportement cynique de la propagande donne aussi le jour à l'esthétisme de ses auteurs. Ce sont les représentants modernes du principe de l'Art pour l'art, et l'entêtement avec lequel ils jouent avec les sentiments de l'âme est le retournement du désespoir, qui a poussé les artistes de la fin du XIX ${ }^{\mathrm{e}}$ siècle à chercher refuge [...] dans le bateau ivre de l'art, comme dans une arche [...]. L'esthétisme des virtuoses de la propagande a un impact sur leur œuvre. Beaucoup de mesures prises par la propagande totalitaire ont pour seul et unique objectif d'exercer une fascination esthétique [...]. L'esthétisation de la propagande provoque l'anesthésie des masses. Le déluge des images les rend insensibles envers la signification réelle des événements que la propagande déforme, de telle façon que la volonté de prendre ces images d'assaut est étouffée en eux. Autrement dit : en tant que la propagande totalitaire transforme des phénomènes qui pourraient être l'objet d'une analyse en objets d'une contemplation esthétique, elle fait en sorte que ces phénomènes se consolident et restent à l'abri de l'analyse. Les masses arrangées sont des figures desquelles on ne parvient pas à détourner le regard ${ }^{18}$.

17. — Siegfried Kracauer, " Exposé Masse und Propaganda », in : Id., Totalitäre Propaganda, op. cit., p. 235.

18. - Sauf mention contraire, nous traduisons. S. Kracauer, Totalitäre Propaganda, op. cit., p. 64. «Derselbe Nihilismus, der das zynische Verhalten der Propaganda bedingt, erzeugt auch den Ästhetizismus ihrer Urheber. Sie sind die modernen Vertreter des l'art-pour-l'art-Prinzips und die Verstocktheit, mit der sie auf der Seele spielen ist der Umschlag der Verzweiflung, aus der heraus die Fin-de-siècle Künstler sich und das Ihre ins trunkene Schiff der Kunst wie in eine Arche retteten [...]. Das Ästhetentum der Propaganda-Virtuosen wirkt sich in ihren Schöpfungen aus. Viele Maßnahmen der totalitären Propaganda sind einzig und allein darauf berechnet, ästhetisch zu faszinieren [...]. Das Ästhetisieren der Propaganda bezweckt die Anästhetisierung der Massen. Der Sturm der Bilder macht sie unempfindlich gegen die eigentliche Bedeutung des von den Bildern verstellten Geschehens, so dass in ihnen der Drang erlischt, diese Bilder zu stürmen. Anders ausgedrückt : indem die totalitäre Propaganda gewisse, der Analyse zugängliche Phänomene in Gegenstände der ästhetischen Betrachtung überführt, sorgt sie dafür, dass sich die betreffenden Phänomene verfestigen und vor der Analyse bewahrt bleiben. Faschistische oder 
Cette notion de « regard » montre bien que dans le nazisme, le peuple devient un spectacle, et que la notion d'image, bien que non thématisée en tant que telle, est bien centrale dans l'approche qu'a Kracauer du totalitarisme. Kracauer renvoie ici surtout au Thingspiele et aux grandes parades du régime, qui sont aussi des spectacles visuels, mais même s'il ne cite pas Leni Riefenstahl, le Triomphe de la volonté apparait comme une confirmation éclatante de cette thèse d'une préparation artistique des masses : un but de ce film semble être la figuration de cette communauté du peuple, représentée comme puissante, revitalisée, sous l'aspect d'un gigantesque " ornement de la masse », en particulier dans la scène du « front du travail ». Le Führer omniprésent apparaît comme une figure charismatique liée organiquement à cette masse du peuple dont il incarne l'unité. Le film de Riefenstahl confirme également l'intuition de Kracauer dans le texte l'« Ornement de la masse » de 1927, qui expliquait que le retour de l'ornement, sous la forme d'une ornementalisation des corps et des masses témoignait d'un retour du mythe et d'une crise de la rationalisation ${ }^{19}$. Le régime nazi est donc selon Kracauer consubstantiel à sa propagande, qui consiste essentiellement en des images, dans lesquelles le contenu idéologique est secondaire. Il s'agit en effet de produire une belle apparence, et dans cette tradition esthétique que Kracauer appelle " l'art pour l'art », le contenu est tout à fait accessoire. Comme l'expliquait déjà Friedrich Schiller, un des fondateurs de cette esthétique de l'autonomie, la forme efface le contenu, qu'il soit sensible ou moral : la belle apparence (schöner Schein) ne renvoie qu'à elle-même. Dans les années 1970 et 1980, des historiens comme Peter Reichel, ont repris cette approche de Benjamin et Kracauer ${ }^{20}$. Dans ce type d'analyse, on tend à présupposer que si le public est consentant, c'est parce qu'il est séduit, manipulé, et dans Totalitäre Propaganda, Kracauer expose les mécanismes psychologiques de cette manipulation qui s'adresse à ce qu'il appelle la structure psycho-physique des individus : la terreur contribue ainsi à cette déstabilisation de l'équilibre psychique en rendant les individus réceptifs aux images produites par le régime.

\section{Image et idéologie}

Une limite évidente de cette approche axée sur la séduction et la manipulation psychologique est qu'elle peut conduire à négliger la

nationalsozialistische Massenaufgebote sind Gestaltungen, von denen man den Blick nicht wenden kann ».

19. - Siegfried Kracauer, «L'ornement de la masse », in : Id., L'ornement de la masse : essais sur la modernité weimarienne (traduit de l'allemand par Sabine Cornille ; préface d'Olivier Agard ; texte édité par Olivier Agard et Philippe Despoix), Paris, la Découverte, 2008, p. 60-71.

20. - Peter Reichel, La fascination du nazisme, Paris, Odile Jacob, 1997. 
conviction idéologique des acteurs, et les contenus idéologiques euxmêmes, comme si ceux-ci étaient indifférents, alors que les plus grands crimes du national-socialisme comme l'extermination des Juifs européens et la guerre à l'Est se sont faits au nom d'une idéologie bien définie. Johann Chapoutot et d'autres ont montré qu'il fallait absolument prendre en compte ces convictions idéologiques des acteurs, et que l'on a trop souvent négligé ce facteur. Chapoutot, tout en rendant hommage à Reichel, développe l'idée que le national-socialisme n'est pas seulement une " esthétique », mais aussi une " éthique » (avec beaucoup de guillemets) ${ }^{21}$. Un autre problème lié à celui-ci est que, lorsque l'on parle d'esthétisation du politique, la communauté du peuple apparaît comme une image sublimée, un spectacle séduisant, un simulacre. Or, si les historiens semblent unanimes pour considérer que l'idée de Volksgemeinschaft est un élément central de la propagande nazie, même si ce n'est pas eux qui l'ont inventée et mise en circulation, et s'ils sont aussi d'accord pour dire que la réalité de la société nazie n'a jamais correspondu à cet idéal, ils s'intéressent de plus en plus aux pratiques sociales d'inclusion ou d'exclusion qui ont donné pour beaucoup d'Allemands un début de réalité à cette idée, et expliquent donc leur consentement à l'idéologie du régime, et leur participation à son fonctionnement. On pourrait donc s'étonner qu'un fin critique de l'idéologie comme Kracauer considère à ce point les contenus idéologiques comme secondaires, allant jusqu'à écrire : « Le concept national-socialiste de peuple est un simple emballage sans contenu $22 »$. Il est frappant de voir qu'alors que quelques années plus tard, Hannah Arendt fera en revanche de l'idéologie le principe qui explique la perte de la réalité et du monde commun, Kracauer ne s'intéresse pratiquement pas à l'idéologie national-socialiste en tant que telle, et évoque à peine les concepts de race, de Volksgemeinschaft, de Führer, se contentant d'expliquer que cette idéologie est régressive, antilibérale et antidémocratique. Il parle aussi très peu de l'antisémitisme, alors que quelques années auparavant il avait écrit : « l'antisémitisme est en réalité un élément central du mouvement ${ }^{23} \gg$. Kracauer n'ignore certainement pas l'existence de cet arrière-plan idéologique ${ }^{24}$, mais pour lui, ce n'est pas l'idéologie qui fait la spécificité du régime en tant que système totalitaire et qui en constitue

21. - Johann Chapoutot, La loi du sang : penser et agir en nazi, Paris, Gallimard, 2014, p. 20.

22. - S. Kracauer, Die totalitäre Propaganda, op. cit., p. 57.

23. - Siegfried Kracauer, « Die deutschen Bevölkerungsschichten und der Nationalsozialismus ", in: Id., Essays, Feuilletons, Rezensionen, 1932-1965 (herausgegeben von Inka Mülder-Bach, unter Mitarbeit von Sabine Biebl) [Werke; 5.4], Berlin, Suhrkamp, 2011, p. 444

24. - Il connaissait par le biais de sa tante et de sa mère, qui étaient restées en Allemagne (et mourront en déportation) la réalité de la politique antisémite de Hitler. 
le moteur profond. Elle n'est qu'un prétexte à une volonté destructrice. À la base de la belle image de la communauté du peuple, il y a surtout une pure volonté de pouvoir, et c'est pour cette raison que Kracauer recourt au terme de «nihilisme » :

Une volonté nihiliste de pouvoir est [...] l'impulsion décisive, à laquelle obéissent les cliques fascistes et nazies : il est par ailleurs clair que cette impulsion ne peut pas être considérée simplement comme une idéologie ou comme produit dérivé de forces sociales et qu'elle doit être appréhendée dans sa cohérence ${ }^{25}$.

Ce terme de nihilisme peut prêter à confusion. À la même époque, ce terme est popularisé par Hermann Rauschning dans son livre $L a$ révolution du nihilisme (Die Revolution des Nihilismus. Kulisse und Wirklichkeit im Dritten Reich, 1938), mais il faut bien comprendre que Kracauer n'adhère pas à la thèse conservatrice, qui sous-tend en général l'usage de ce terme, par lequel on implique que les valeurs traditionnelles auraient été le meilleur antidote contre la révolution national-socialiste, ce qui n'est pas du tout le diagnostic de Kracauer. Tous ses textes sur le cinéma montrent en effet que c'est au contraire une certaine dynamique critique, post-religieuse, post-ontologique de la modernité qui permettra pour lui de sortir de cette irréalité nazie, et que la modernité ne saurait être définie comme nihilisme.

Si la propagande véhicule des contenus idéologiques, ceux-ci ne sont pas l'élément moteur, même si par ailleurs, objectivement, le nazisme rejoint les intérêts du capitalisme de monopole (Monopolkapitalismus), terme que Kracauer emploie à deux ou trois reprises dans Totalitäre Propaganda, rejoignant les analyses en vigueur au sein de l'Institut für Sozialforschung. À bon droit, Kracauer pouvait considérer qu'il faisait partie de ceux qui avaient perçu en Allemagne une menace de décivilisation, une violence criminelle irréductible à de simples intérêts socio-économiques. Dans une lettre de 1930 à Adorno, au moment des élections législatives qui virent une poussée des nazis, il écrit : «Une malédiction plane sur ce pays, et je sais exactement qu'il ne s'agit pas seulement du capitalisme. Ce n'est en aucun cas pour de seules raisons économiques que celui-ci peut prendre une forme aussi bestiale $26 »$.

25. - S. Kracauer, Totalitäre Propaganda, op. cit., p. 29. « Nihilistischer Machtwille [...] ist der entscheidende Impuls, dem die faschistischen und nationalsozialistischen Cliquen gehorchen. Dieser Impuls, das steht ferner fest, lässt sich nicht ohne weiteres als Ideologie überhaupt oder Derivat gesellschaftlicher Kräfte begreifen und muss daher einstweilen in seiner Verschlossenheit hingenommen werden ».

26. - Siegfried Kracauer, Lettre à Adorno du 24 août 1930, in : T.W. Adorno-Siegfried Kracauer, Briefwechsel 1923-1966, Suhrkamp, Frankfurt a.M., 2008, p. 246. «Es waltet ein Verhängnis über diesem Land und ich weiss genau, dass es nicht nur der Kapitalimus ist. Dass dieser so bestialisch werden kann, hat keineswegs ökonomische Gründe ». 
Cette lettre à Adorno est significative du refus de faire du nazisme un simple dérivé du libéralisme et un aboutissement du règne capitaliste de la marchandise, et de ce point de vue, Kracauer entrait en porte-à-faux avec Adorno et Horkheimer, qui, du moins dans les années 1930, insistaient plutôt sur cette continuité. Selon Kracauer, même si l'image manipulatrice existe aussi dans un contexte démocratique, elle n'abolit pas totalement la réalité. C'est notamment ce qu'il explique à Paul Lazarsfeld dans une lettre de 1942 : «Alors que les démocraties utilisent jusqu'à un certain point des effets de mise en scène, la perpétuation du système nazi dépend de leur mise en œuvre. On peut imaginer une démocratie qui renoncerait à de tels effets : le royaume nazi ne pourrait exister sans ces manœuvres de la propagande totalitaire 27 ».

En ce sens, on peut dire que Kracauer utilise le terme de « totalitaire » dans un sens spécifique, et pas seulement comme un synonyme de « autoritaire » ou « fasciste » ou « nazi ». Le nazisme abolit totalement la perception commune d'un monde matériel, à l'intérieur duquel les conflits peuvent s'exprimer, parce qu'on est au moins d'accord sur les termes du désaccord, qui oppose alors des individus et des intérêts réels. Dans une analyse qui reste stimulante pour aujourd'hui, Kracauer explique qu'il ne s'agit d'ailleurs pas de remplacer une réalité par une autre, ou de recouvrir le monde commun par l'idéologie, mais de décrédibiliser l'idée même de vérité, de saper toute confiance dans l'idée d'une vérité : c'est la stratégie du Spiegelreflex qui consiste à attribuer ses propres turpitudes à l'ennemi, si bien que le public ne sait plus du tout à quoi s'en tenir. C'est aujourd'hui une stratégie analogue qui est en œuvre dans les Fake News ou «infox ».

\section{La manipulation de l'image}

Par la suite, les textes américains analysent de façon plus précise les mécanismes par lesquels se produit cette abolition de la réalité. Un point commun avec la Propagande totalitaire est que Kracauer ne s'intéresse pas prioritairement aux contenus idéologiques. Il ne procède pas à une critique idéologique de la propagande ; la forme visuelle de celle-ci est pour lui ce qui importe, et il y a une bonne raison à cela : « Dans les bandes d'actualités de guerre nazies, les images prévalent sur le commentaire. Ce primat de l'élément visuel est un moyen tout à

27. - Siegfried Kracauer, lettre à Paul Lazarsfeld du 23.11 .1942 (citée in: Johannes von Moltke, The curious humanist : Siegfried Kracauer in America, Oakland, University of California press, 2016, p. 77). «Whereas the democracies utilize at a certain extent staged effects, the maintenance of the Nazi system depends upon their exploitation. It is possible to imagine a democracy renouncing any such effects: the Nazi realm could not exist without these manouvers of totalitarian propaganda ». 
fait significatif et utilisé de façon consciente 28 ». Le lien avec la théorie du cinéma comme média réaliste apparaît maintenant comme fondamental : Kracauer montre que la propagande met en œuvre des procédés qui pourraient tout autant êtes mis au service de la connaissance de la réalité. C'est là un point de divergence majeur avec Adorno : si le nazisme séduit par l'image, celle-ci n'est pas intrinsèquement manipulatrice. La sortie de l'irréalité passe donc aussi par l'image, car l'image (et plus particulièrement l'image photographique) n'est pas nécessairement irréelle ou trompeuse. Kracauer donne, dans ces textes, le nom de « Sham Reality » ${ }^{29}$ ou « Svastika world ${ }^{30}$ » à ce qu'il appelait « pseudoréalité » dans Totalitäre Propaganda. Il souligne en premier lieu que la représentation de la réalité dans les actualités cinématographique donne le sentiment d'une maîtrise totale du temps et de l'espace, grâce aux nombreux plans panoramiques et à la mobilité de la caméra. Selon Kracauer, le montage contribue aussi à ce sentiment de maîtrise de la réalité matérielle. Il souligne que les films qui ont été montés à partir des actualités cinématographiques mettent en œuvre de façon massive toutes les possibilités techniques du montage. À propos des auteurs des actualités, il écrit : «Il est difficile de trouver le moindre procédé de montage qu'ils n'aient exploité, et il existe plusieurs moyens de présentation dont ils ont porté l'efficacité à une ampleur jusqu'ici inconnue ${ }^{31} »$. Il est aujourd'hui bien connu qu'on retrouve ce même usage du montage, en partie inspiré de l'avant-garde soviétique, dans les films de Leni Riefenstahl, qui tournait énormément d'images. Kracauer insiste sur le rôle des ellipses qui abolissent le sentiment de réalité, « donnant au public un sentiment de facilité de l'accomplissement et renforçant l'impression d'une guerre-éclair allemande indomptable 32 ». Kracauer montre par ailleurs comment l'image exploite l'état de conscience diminué du spectateur distrait, en mobilisant son système nerveux. Dans Le Triomphe de la volonté, les mouvements de la caméra pilotent la perception du spectateur :

Le mouvement produit par des techniques cinématographiques appuie celui des objets. Les panoramiques, les travellings, les plongées montantes ou descendantes sont constants - de manière à ce que le spectateur ne voie pas seulement défiler un monde enfiévré, mais s'y

28. - Siegfried Kracauer, « Die Eroberung Europas auf der Leinwand », in: $I d$., Studien zu Massenmedien und Propaganda, op. cit., p. 444.

29. - Par exemple dans la lettre déjà évoquée à Lazarsfeld du 23.11.1942.

30. — « Le monde de la svastika », in : S. Kracauer, « La propagande et le film de guerre nazi ", in : Id., De Caligari à Hitler : une histoire psychologique du cinéma allemand (traduit de l'anglais par Claude B. Levenson), Lausanne, Paris, l'Âge d'homme, 2009, p. 318.

31. - Ibid., p. 314.

32. - Ibid., p. $315 s q$. 
sente aussi enraciné. La caméra omniprésente l'oblige à suivre les routes les plus fantastiques, et le montage aide à l'y conduire ${ }^{33}$.

La propagande stimule les «nerfs moteurs », en particulier par le biais de la musique ${ }^{34}$, et on a ici l'illustration concrète d'une action psychophysique de la propagande sur les sujets, déjà présent dans Totalitäre Propaganda. Toujours dans la continuité de Totalitäre Propaganda, Kracauer évoque l'image de la communauté du peuple telle qu'elle est fabriquée par Leni Riefenstahl dans Triumph des Willens : « [...] dans le Triomphe de la volonté des ornements spectaculaires formés de foules excitées et de drapeaux à la Svastika claquant au vent servaient à donner une substance à la prétendue collectivité que les leaders nazis avaient créée ${ }^{35}$ ». À cette pseudo-communauté, Kracauer oppose la collectivité que met en scène Sergueï Eisenstein dans Le Cuirassé Potemkine, et qui est composée de personnes réelles. Comme dans Totalitäre Propaganda, Kracauer considère que cette apparence cultivée pour elle-même marque « le triomphe d'une volonté nihiliste ${ }^{36} »$.

\section{Image et réalité}

Mais l'intérêt de ces textes est comme on l'a dit la façon dont ils s'inscrivent dans une réflexion sur le médium cinématographique. Tout se passe comme si la réflexion sur l'image de propagande était l'occasion d'explorer les potentialités positives du cinéma qui seront présentées dans Theorie des Films, et qui le sont déjà en partie dans le manuscrit de 1940 (que faute de place, on laissera ici de côté). Le cinéma doit son efficacité comme moyen de propagande précisément en vertu de son lien à la réalité, que Kracauer entend au sens de réalité matérielle, celle du monde physique qui se donne à voir dans la photographie. En ce sens, la propagande utilise et détourne à la fois le potentiel de connaissance de cette réalité matérielle qui est celui du cinéma. En effet, du fait qu'il est un dispositif technique d'enregistrement, le cinéma donne accès à une réalité non-intentionnelle qui ne pouvait pas être aperçue auparavant.

Par ailleurs, le cinéma correspond à un âge où ni la réalité ni la subjectivité n'ont plus de centre, et ne peuvent plus être appréhendées comme des totalités. Chez Kracauer, cette absence d'un centre n'est pas, dans l'œuvre tardive, l'objet d'une sorte de déploration. Elle est le principe d'une connaissance nouvelle de la réalité et ouvre la possibilité de fonder un monde humain qui sera véritablement un monde commun. En effet, la diminution de la conscience n'est pas, en soi, un phénomène

33. - Ibid., p. 345 .

34. - S. Kracauer, « Die Eroberung Europas auf der Leinwand », op. cit., p. 449.

35. - S. Kracauer, « La propagande et le film de guerre nazi », op. cit., p. 329.

36. — Ibid., p. 346. 
négatif, ou du moins, nécessairement régressif. Elle n'est pas seulement propre à la masse séduite dans la propagande, mais caractérisera dans la Théorie du film le spectateur de cinéma en général : «Chez le spectateur de films, le moi, en tant qu'agent principal des pensées et des décisions, renonce à son pouvoir de contrôle ${ }^{37}$ ». Kracauer ajoute : « Aussi le film tend-il à émousser la conscience du spectateur $»^{38}$.

De même, la motorisation de la perception guidée par la caméra n'est pas propre au cinéma de propagande de Riefenstahl, mais caractérise le cinéma en tant que medium, comme on pourra le lire dans la Théorie $d u$ film $^{39}$ : «Le spectateur devra, par exemple, s'identifier à la caméra effectuant un panoramique vertical ou un travelling, dont le but est d'attirer son attention sur des objets qui peuvent être aussi bien fixes qu'animés ». Le cinéma s'adresse ainsi à une subjectivité ouverte, plastique, exposée au chaos du monde moderne, celle du Spectator ${ }^{40}$, qui n'est plus le sujet de l'idéalisme ou de la société bourgeoise.

Cet état de flottement et d'ouverture, dans lequel nous place le cinéma, est justement celui qui permet la connaissance du monde matériel. En effet, le film est comme un rêve, mais ce rêve a deux directions, l'une tournée vers l'objet, et l'autre vers l'intérieur : «L'immersion, proche de la transe, dans un plan ou dans une succession de plans, peut à tout moment céder la place à une rêverie qui se dégage progressivement des images qui l'ont suscitée ${ }^{41} »$. Cette combinaison de « self-abandonment » et de «self-absorption » est ce qui rend possible l'accès au monde physique et une connaissance sans préjugé de la réalité matérielle.

En tant que moyen de connaissance d'une réalité matérielle postsymbolique qui devient visible avec le désenchantement du monde, le cinéma a une fonction éminemment politique, qu'il a par ailleurs en tant qu'expression et moteur d'une dynamique sociologique de démocratisation. Le centre de gravité sociologique du public du cinéma est la classe moyenne, qui voit se rejoindre le prolétariat et le Mittelstand, à rebours des hiérarchisations traditionnelles. Dans ses textes weimariens, Kracauer avait déjà indiqué que le divertissement avait le mérite de la " sincérité » (Aufrichtigkeit), dans la mesure où il contribuait à liquider l'héritage bourgeois et idéaliste. La Zerstreuung avait donc un aspect émancipateur. Dans La Propagande totalitaire, Kracauer dit explicitement que cette conscience amoindrie de la masse, qui facilite le travail de la propagande, aurait tout autant pu être mise au service d'une révolution politique : «Il est certain qu'il se produit également au

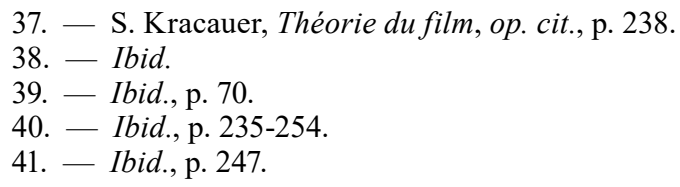


sein de la masse révolutionnaire une diminution de la conscience. Mais cet effacement a une fonction positive dans la mesure où il renforce jusqu'à un certain point l'intérêt révolutionnaire, dirigé contre les excès de l'individualisme ${ }^{42} »$.

Dans Totalitäre Propaganda, la réalité occultée par la pseudo-réalité nazie est celle de la confrontation sociale, mais dans les travaux des années 1940-60 Kracauer insiste sur le fait que cette confrontation n'est possible que sur la base d'une perception partagée d'un monde matériel commun, que le cinéma contribue à rendre possible. Pour Kracauer, c'est cette perception qui manquait traditionnellement en Allemagne dans les années 1920. À cet égard il est intéressant de mettre en regard la Propagande totalitaire et les textes politiques des années 1920 et 1930. Un élément central du diagnostic de Kracauer sur la République de Weimar est déjà l'irréalité comme absence d'un monde commun. L'affrontement politique prend dans la République de Weimar la forme d'un affrontement de visions du monde, de drapeaux de symboles, dans lesquels la réalité matérielle des hommes est tout à fait secondaire. Même le maillot de bain devient le prétexte à développer une vision du monde : « Chez nous, on peut à peine acheter une brosse à dents sans recevoir une vision du monde en cadeau. C'est donc en raison de leur vision du monde que $\mathrm{M}$. Koch et ses disciples déclarent la guerre au maillot de bain. Le maillot de bain, dit M. Koch, est une superstition qu'il faut combattre ${ }^{43}$ ». À propos d'un débat entre un représentant de l'« Ordre de la jeunesse allemand » (de tendance nationale libérale) et un étudiant social-démocrate, Kracauer montre l'impossibilité de la confrontation démocratique des discours en Allemagne : "Comme souvent en Allemagne, le dialogue s'est peu à peu élevé dans les hautes sphères des visions du monde, et on a fini par assister à l'opposition entre l'idée d'un État populaire fondé sur l'autogestion et celle de l'économie socialiste planifiée $44 »$. Ce diagnostic d'irréalité explique la nostalgie d'une communauté naturelle, que l'on perçoit parfois chez Kracauer, en particulier quand il parle de la France. Dans la lettre déjà évoquée à Adorno, dans laquelle il s'inquiétait de l'existence d'une dynamique mortifère en Allemagne, il écrit à propos de la France, où il se trouve alors : « En France, où il y a certainement beaucoup de chose critiquables, je retrouve toujours ce qui chez nous a été détruit : la décence élémentaire, la nature bienveillante et entière,

42. - S. Kracauer, Totalitäre Propaganda, op. cit., p. 84.

43. - Siegfried Kracauer, «Lutte contre le maillot de bain », in : Id., Politique au jour le jour : 1930-1933, (traduit de l'allemand par Jean Quétier; avec la collaboration de Katrin Heydenreich ; préface de Jean Quétier), Paris, Éditions de la Maison des sciences de l'homme, 2017, p. 51.

44. - Siegfried Kracauer, «En dehors de l'université - discussion d'étudiants », in : Id., Politique au jour le jour, op. cit., p. 85. 
et avec elle, cette confiance des hommes les uns dans les autres ${ }^{45} \gg$. En Allemagne, l'espace public est envahi par des idéologies et des mythes qui empêchent le véritable dialogue, celui-ci n'étant possible que sur la base d'une perception partagée d'un monde commun. Ce sentiment de l'absence d'une réalité commune, est aussi la raison de l'intérêt, qui peut a postériori paraître étrange, que Kracauer montre pour l'expérience des « camps de travail », inspirés par Eugen Rostenstock 46 et Hans Dehmel d'une part, et par certains courants de la Jugendbewegung d'autre part ${ }^{47}$. Officiellement, l'idée qui préside à ces camps est de rassembler des jeunes gens de toutes origines sociales. En 1931, l'ordonnance sur le service du travail (sur la base du volontariat) du chancelier Brüning semble annoncer la généralisation de cette expérience. Kracauer, racontant une visite dans un de ces camps, refuse certes de les considérer comme les cellules d'une « communauté du peuple », ce qui est selon lui une illusion romantique et une naïveté politique. Il explique toutefois que les Arbeitslager pourraient dans certaines conditions non pas fournir un modèle de société, mais au moins aider à créer un espace politique en favorisant la confrontation des points de vue ${ }^{48}$. Il s'agit donc pour Kracauer, par cette rencontre non plus entre idéologies mais entre personnes, de poser les bases d'une perception commune qui est une condition pré-politique pour l'existence d'une communauté politique, qui doit être incarnée matériellement, pour développer une conflictualité positive. Cette perception ne peut être commune que parce qu'elle est ancrée dans la réalité matérielle, où les conflits deviennent concrets et opposent des individus réels, et le cinéma apparaît donc comme le medium d'une éducation esthétique post-idéaliste, un apprentissage de la réalité.

Cette perception commune n'est pas donnée de façon immédiate, mais suppose un travail herméneutique et critique du sujet, notamment parce que l'image photographique est toujours hybride. Elle a un rapport à la réalité matérielle, que n'avaient pas les arts visuels traditionnels, mais elle est également formée : l'image est toujours une lecture de la réalité à partir de laquelle le spectateur élabore sa propre lecture.

D’une certaine façon, la réalité matérielle n'est qu'un point de départ pour cette communauté humaine à construire, mais elle en est la base

45. - Siegfried Kracauer, Lettre à Adorno du 24 août 1930, in : T. W. Adorno-S. Kracauer, Briefwechsel, op. cit., p. 247. « Ich bemerke immer wieder in Frankreich, an dem es doch gewiss viel zu kritisieren gibt, was alles bei uns zerstört ist: der primitive Anstand, die ganze gute Natur, und mit ihr jedes Vertrauen der Menschen ineinander ».

46. - Cf. Eugen Rosenstock-Huessy, Das Arbeitslager: Berichte aus Schlesien von Arbeitern, Bauern, Studenten, Jena, Diederichs, 1931. Voir également les fragments autobiographiques : Ja und Nein, Heidelberg, Schneider, 1968.

47. - Siegfried Kracauer, « À propos des camps de travail », in : Id., Politique au jour le jour, op. cit., p. 147-157.

48. - Ibid., p. 155. 
nécessaire. C'est cette idée d'une communauté humaine, qu'à tort ou à raison, Kracauer voit à l'œuvre dans l'exposition « Family of man » (organisée par Edward Steichen pour le Musée d'art moderne de New York en 1955). C'est en effet sur l'évocation de cette exposition que s'achève Theory of film.

\section{Le retour du réel}

Un des points les plus intéressants des analyses de Kracauer sur la propagande me paraît être l'idée que le lien à la réalité matérielle enregistrée par un dispositif technique existe également dans l'image de propagande, à rebours et à l'insu des intentions de celle-ci : il y a toujours dans l'image quelque chose qui échappe au contrôle et à l'intention idéologique. Même dans les images manipulatrices de la propagande, il y a des cas où cette limite devient manifeste. Kracauer donne l'exemple des images célèbres de Hitler en visite à Paris le 23 juin 1940. Il attire l'attention sur le fait qu'Hitler évolue dans une ville vide et que ces images qui sont supposées symboliser le contrôle total de la réalité donnent en réalité à voir le nihilisme de la volonté de pouvoir nazie :

Le Führer visite la capitale européenne conquise - mais en est-il réellement l'hôte ? Paris est aussi tranquille que grave. À part quelques agents de police, un ouvrier et un prêtre solitaire qui se hâte hors du champ de vision, il n'y pas une âme au Trocadéro, à l'Etoile, sur la vaste Concorde, à l'Opéra et à la Madeleine, pas une âme pour saluer le dictateur si accoutumé aux foules applaudissantes. Tandis qu'il inspecte Paris, Paris lui-même ferme les yeux et s'éloigne. La vision touchante de cette cité fantôme désertée qui autrefois vibrait de vie fiévreuse reflète le vide du propre noyau du système nazi. La propagande nazie avait construit une pseudo-réalité rayonnante de mille couleurs, mais en même temps, elle vidait Paris, le sanctuaire de la civilisation. Ces couleurs pouvaient difficilement masquer son propre vide ${ }^{49}$.

Depuis quelques années, certains historiens français insistent eux aussi sur cette possibilité d'une double lecture des images de propagande. Sylvie Lindeperg a ainsi montré comment dans le tristement célèbre film sur Theresienstadt ${ }^{50}$, on trouve de nombreux indices du caractère fictif de la pseudo réalité mise en scène dans ce film ${ }^{51}$. Les personnes filmées et contraintes de jouer un rôle cassent l'illusion par exemple en regardant

49. - S. Kracauer, «La propagande et le film de guerre nazi », op. cit., p. 351 sq.

50. - Le film est connu sous le titre Theresienstadt. Der Führer schenkt den Juden eine Stadt, mais en réalité, il était intitulé : Theresienstadt. Ein Dokumentarfilm aus dem jüdischen Siedlungsgebiet.

51. - Sylvie Lindeperg, La voie des images : quatre histoires de tournage au printemps-été 1944, Lagrasse, Verdier, 2012, p. 103-141. 
directement la caméra ou en se masquant le visage. Il y a, à cet égard, un « double jeu » du cinéma ${ }^{52}$, qui peut évoquer une phrase clé de la conclusion de la Théorie du film 53 : « le film rend visible ce que nous n'avions pas vu, et que peut-être ne pouvions pas voir, avant qu'il soit là ${ }^{54} \gg . \mathrm{Ce}$ n'est pas un hasard si Kracauer conclut son texte sur cette perspective d'une autodestruction de la propagande, parce qu'elle illustre l'essence du cinéma. Dans le film de propagande, cet effet de réel est involontaire, mais ce dispositif peut tout à fait être utilisé de façon consciente, et le cinéma atteint le maximum de son pouvoir de connaissance. C'est ainsi que dans Hôtel des invalides (1951), Franju, sous prétexte d'un documentaire sur le musée de la guerre du palais des Invalides, déconstruit - par toute une série d'éléments subtilement dissonants - le discours patriotique et héroïque propagé par le musée et son guide. Devant la caméra de Franju, « l'armure au lion » de François $\mathrm{I}^{\mathrm{er}}$ devient ainsi un spectacle de cauchemar digne d'un film d'épouvante (ce plan fait partie des images que Kracauer a retenues pour illustrer sa Théorie du film). Dans ce film aux fausses allures de propagande institutionnelle, Franju met en scène la capacité de l'image de cinéma à montrer autre chose que ce qu'elle prétend montrer en tant qu'image intentionnelle, et, au terme d'un processus critique, de nous faire approcher la réalité matérielle.

52. - Ibid., p. 143.

53. - Ibid.

54. - S. Kracauer, Théorie du film, op. cit., p. 460. 